\title{
An approximate method for controlling delay intervals in subject-paced group learning experiments
}

ERNST Z. ROTHKOPF

BELL TELEPHONE LABORATORIES, MURRAY HILL, NEW JERSEY

A simple technique is described for imposing E-controlled time intervals in group experiments in which some phases are paced by $S$. This technique involves a line-marking task under the control of a digital clock display. The display is also equipped with a random element. Fairly accurately timed intervals have been obtained with this technique in two learning experiments.

Group techniques have been used in learning studies in which Ss' activities are paced by $\mathrm{E}$ (e.g., Bilodeau et al, 1963; Saltz \& Myers, 1955; Rothkopf, 1958) and in studies in which each $\mathrm{S}$ proceeds through all phases of the experiment at his own rate (Rothkopf \& Coke, $1963,1966)$. The latter procedure is of particular interest in programmed instruction research (e.g., Glaser \& Reynolds, 1964) and in studies in which $S^{\prime}$ 's pace is a dependent variable (e.g. , Rothkopf, 1966). Group methods, while economical ways for collecting data, pose special problems when some phases of an experiment are to be paced by $S$, and others must remain under $E$ control. This may occur in studies which require the experimental manipulation of time periods within an experimental session, e.g., retention intervals, interpractice intervals, and so on. By illustration, in a learning experiment that has an S-paced acquisition, an Econtrolled time delay, and an S-paced retention test phase, E's technical resources may be taxed because Ss, operating at their own pace in acquisition, enter the delay phase at different times.

We have found a simple, approximate method for controlling variable time intervals under S-paced conditions. The technique involved a digital clock equipped with a random element. In our case, this took the form of a film strip projector stepped by two Hunter Decade timers. A "time" number projected on the screen, was changed every $t$ seconds. Whenever the number was changed it was incremented by one. The random element of this digital display was a bar which appeared on the film strip either just above or below the "time" number and which had been assigned to these numbers according to a random schedule.

During the S-paced phases of the experiments the projected digital clock was used by Ss to record start and finish times. When $\mathrm{S}$ entered the controlled delay phase, he opened an envelope containing instructions which directed him to print consonants on the first line of an especially mimeographed worksheet and to start print- ing on a new line whenever the digits of the time displayed changed. The work sheet was divided into two identical columns by a vertical line. The purpose of the random element, the bar, was to discourage $S s$ from anticipating the actions of the clock and performing the printing operation in advance. This was done by requesting Ss to print the consonants in the column on the left side of the page if the bar was above the time display and on the right side if the bar was below the number. When $S$ reached the last row of the worksheet, he found directions to go on to the nextphase of the experimental task. The controlled interval was thus determined by: (a) the time required to open the envelopes in which the experimental materials of each phase were kept and to read the brief directions; (b) by the rate at which the digital time display was changed; and (c) by the number of rows (lines) provided on each S's worksheet. The last factor makes it possible to determine different delay times for each $\mathrm{S}$ in the experimental group.

The technique has been used to place a delay interval between two self-paced exposures to written experi-

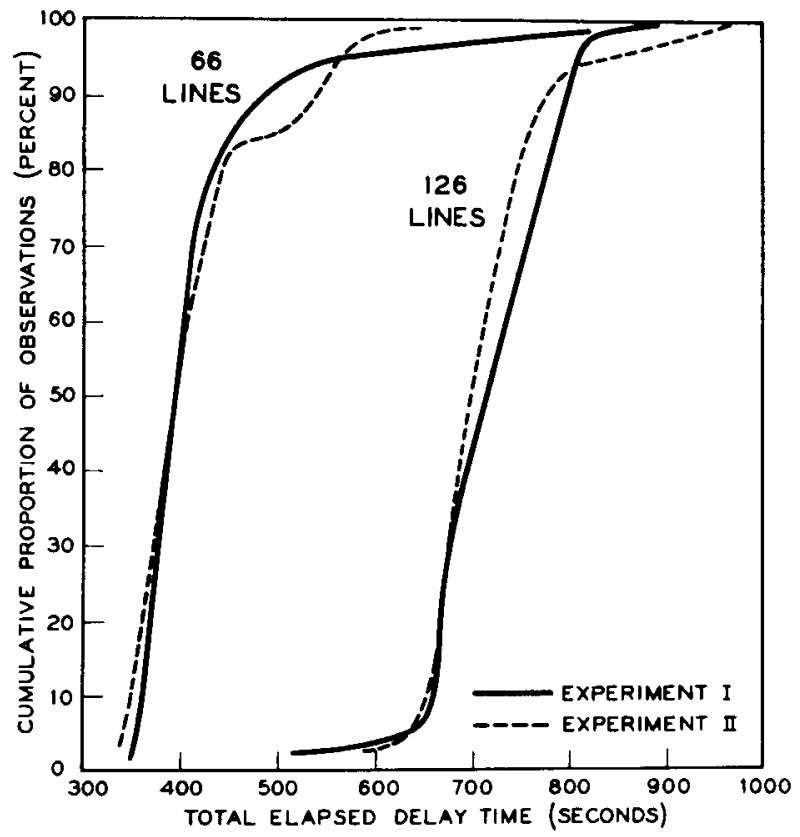

Fig. 1. Cumulative proportion of $\mathrm{Ss}$ who completed the delay period by the end of various time periods (sec.). The two polygons at left were obtained when the 66-line worksheet was used. The two on the right were obtained with the 126-line worksheet. 
mental discourse in two verbal learning studies (Rothkopf \& Coke, submitted for publication). Actual elapsed delay time was recorded for worksheets of 66 and 126 lines in length. The time display was changed every 5 sec. Mean total elapsed delay time for the 66 line form was $426 \mathrm{sec}$. $(\sigma=89.5, \mathrm{~N}=55)$ in Experiment I and 423.4 sec. $(\sigma=75.1, N=52)$ in Experiment II. The time spent in reading directions was $57.4 \mathrm{sec} .(\sigma=23.2)$ and $59.7 \mathrm{sec} .(\sigma=36.3)$ in the two experiments respectively.

Overall $\overline{\mathrm{X}}$ elapsed delay time for the 126-line form was for the two experiments respectively $724.5 \mathrm{sec}$. $(\sigma=66.1, \mathrm{~N}=54)$ and 718.6 sec. $(\sigma=68.6, \mathrm{~N}=46)$. The $\overrightarrow{\mathrm{X}}$ direction reading times in this situation were $57.6 \mathrm{sec}$. $(\sigma=30.8)$ and $57.00 \mathrm{sec} .(\sigma=28.7)$ respectively in the two experiments.

The delay time distributions were somewhat skewed and this limits the usefulness of $\sigma$ as an indicator of accuracy. A more useful impression of the accuracy of the experimental procedure may be obtained by inspecting the cumulative proportion polygons for total delay time in Fig. 1.

Clock-paced line marking with a random element in the clock display appears a simple, approximate way of imposing E-controlled time delays in group experiments in which Ss advance at their own rate. The accuracy which we obtained by this method is less than what is needed for some studies. However, prior practice for Ss in the task and an improved experimental form may add precision to this useful group experimentation technique.

\section{References}

Bilodeau, E. A., Fox, P. W., \& Block, K. A. Stimulated verbal recall and the analysis of sources of recall. J. verbal Learn. verbal Behav., 1963, 2, 422-428.

Reynolds, J. H., \& Glaser, R. Effects of repetition and spaced review upon retention of a complex learning task. J. educ. Psychol., 1964, 55, 297-308.

Rothkopf, E. Z. Stimulus similarity and sequence of stimulus presentation in paired-associate learning. J. exp. Psychol., 1958, $56,114-122$.

Rothkopf,E. Z. Learning from Written Material: An exploration of the control of inspection behavior by test-like events. Amer. Educ. Res. J., 1966, in press.

Rothkopf, E. Z., \& Coke, E. U. Repetition interval and rehearsal method in learning equivalences from written sentences. $J$. verbal Learn. verbal Behav., 1963, 2, 406-416.

Rothkopf, E. Z., \& Coke, E. U. Variations in phrasing, repetition interval, and the recall of Sentence material. J. verbal Learn. verbal Behav., 1966, 5, 86-91.

Rothkopf, E. Z., \& Coke, E. U. Learning from written discourse: Effects of previous exposure on mathemagenic behavior (submitted for publication).

Saltz, E., \& Myers, T. I. A method for group presentation of pairedassociates materials. Paper read at Midwestern Psychological Association, 1955. 